\title{
Usability Evaluation and User Acceptance of Mobile Applications for Saudi Autistic Children
}

\author{
https://doi.org/10.3991/ijim.v15i07.19881 \\ Mohammed Fouad Hajjar, Saad Talal Alharbi, \\ Fahad Mahmoud Ghabban ${ }^{(凶)}$ \\ Taibah University, Medina, Saudi Arabia \\ fghaban@taibahu.edu.sa
}

\begin{abstract}
The evolution of mobile technologies in learning, has been increased the requirements for developing appropriate usability model to fall into the same line with mobile learning applications. Usability of mobile learning applications is a topic that is into research, this paper focuses a tailored designed model called (M-UTUAT) mobile usability model that combines People at the Centre of Mobile Application Development (PACMAD) and the unified theory of acceptance and use of technology (UTAUT) to form a more robust model. As an addition to the usability research, an application (ICT) was developed for children with speech delays and children diagnosed with autism spectrum. The application is a mobile/tablet-based application that developed according to mobile usability measures to get the maximum learning skills and abilities to help autistic children. It has been designed according to the needs of the children specific to their category and skills. The autism spectrum is a broad spectrum with high and low disabilities. The application consists of both visual and audio aids to help children feel comfortable and associate objects more easily with their original meaning and connections. (PECS) is one of the most common techniques that have been clinically proven to be effective in such cases. This application helped an individual with autism spectrum as well as children with speech delays or disabilities to communicate and correlate faster and easily. The application can help so many families by constructing a more communicative path for people with disabilities. The application is innovative that it developed in the Arabic language to serve Arabic learners as most of the autistic applications available serve English learners. Results gathered after questionnaire highlight the usability issues with the (ICT) application. We developed an app to overcome usability issues.
\end{abstract}

Keywords-Usability Evaluation - User Acceptance - Mobile Applications Saudi Autistic Children

\section{Introduction}

With technology advancement nowadays, mobile and wireless technologies are changing our day to day lives. Nowadays, even a mobile phone can recognize facial expressions. This coupled with wireless biosensors which measure attention levels 
make it possible to design a new range of emotion-aware apps. Mobile applications can be beneficial in several domains include education, finance and banking, health, manufacturing, and more.

In the domain of education and health for special needs students, more specifically for children with Autism Spectrum Disorder (ASD), mobile technology would strongly benefit them due to many reasons that discussed in the next chapters. (ASD) uses a wide scope of conditions that are characterized by social deficits, communication difficulties, and repetitive behaviors and to a smaller extent, cognitive delays[1]

(ASD) is a cognitive disorder which is characterized by impaired communication, creative impairment, and sensory sensitivities or a pervasive development disorder[2][3]. Autism affects how a person interacts and relates to other individuals. Thus, it is a lifelong disability. The children diagnosed with (ASD) interact with the world differently from other people[2]. However, due to advancement in technology, portable mobile devices can be used as augmentative and alternative communication (AAC) systems to improve social and communication skills for people with (ASD).

To decide an application's convenience, usability estimations must be connected. The expression "usability" is characterized by the International Organization for Standardization (ISO) 9241-11[4][5] as the degree to which an item can be utilized with adequacy, effectiveness and fulfillment in a predetermined context of use. Adequacy is the exactness with which indicated clients can accomplish their objectives in a specific domain. Efficiency is the measure of resources consumed in connection to the precision and culmination of the objective accomplished. Fulfillment is the solace and agreeableness of the work framework to its clients and other individuals influenced by its utilization.

In Saudi Arabia, specialized centers for mute children and people living with (ASD) are very limited. This presents a problem to families who live in cities or towns far from treatment and training centers. These families may not have received adequate training and support to deal with their children. Moreover, (PECS)'s applications that are available in the market currently are costly. Therefore, one of our aspirations is to develop a free application for (PECS)'s learners. It helped to ease communication between families and individuals living with (ASD). Moreover, the available (PECS) apps do not support the Arabic language. This application only supports Arab and emphasizes the support of verbal communication in local Saudi dialects because of the dominance of using local dialects in clinical settings for speech and language therapy as well as everyday communication in non-formal settings such as at home.

This paper shows the usability assessment of Arabic created application "I Can Talk" was created for kids who have (ASD) (ages 4-12), their teachers, and parents, can assist in helping them use the application. We chose to develop a tailored application because available applications in the market do not address the Saudi kid, we developed our own application with Saudi terms. The developed application is a form of augmentative and alternative communication (AAC), which includes all forms of communication which are used to express thoughts and needs, among other things[6]. (ACC) comprises of special augmentative aids, e.g. pictures and symbol communication boards and electronic devices. These aids assist people to express themselves[7]. 
I Can Talk is made for children who have (ASD), since (ACC) assists individuals who have speech problems, others may also utilize it to learn Arabic.

\section{Materials and Methods}

The aim of this paper is to build a user acceptance testing model that can be used to evaluate mobile applications designed to help children diagnosed with (ASD) to improve their communication and interaction with other people and aids them in expressing themselves and their needs.

Based on the conducted literature review, we believe that the use of mobile applications helped (ASD) children improve their communication and interaction skills. In order to validate that, a new model needs to be developed to help in conducting user acceptance testing focusing on application usability.

The next list illustrates the approach we followed to achieve the study objectives: App Development, Build user acceptance testing model focused on usability, Create a survey based on the built user acceptance testing model and Validate the survey results using Cronbach's alpha.

\subsection{Proposed evaluation model (M-UTUAT)}

The proposed model is shown in Figure 1, it's a combination of UTUAT[8] and PACMAD[9]. We chose to combine both model after the exclusion of the effort expectancy from UTUAT and replacing it with (PACMAD) to get a more robust model while testing our application using the elements in next sections. The proposed (MUTUAT) model relies upon 10 technology acknowledgment hypotheses or models. In particular, in the (M-UTAUT) every single attribute has a critical effect on the applications usability making the model to be used in assistance of assessing the applications usability. 


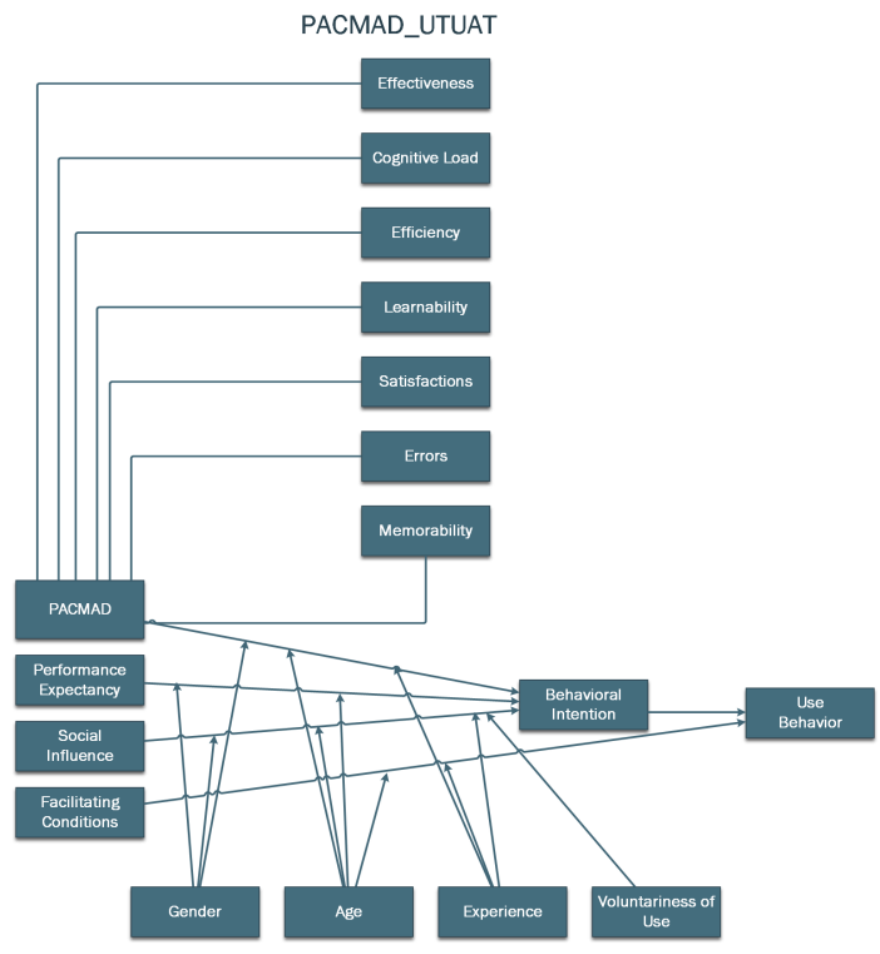

Fig. 1. Proposed Model

\subsection{Cordova architecture}

An application "I Can Talk "was developed using Cordova environment Apache Cordova is a platform for building natively in- stalled mobile applications using common codebase (HTML, CSS and JavaScript)[10]. The characteristic of this state of art technology enable us to use the unified remote laboratory framework: The user interface for Apache Cordova applications is created using common codebase, The web view used by application is the same web- view used by the native operating system, Provides API enables us to access native operating system functionality using JavaScript, Extendable using native plug-in and Support most of the updated operating systems and platforms.

The implementation of Apache Cordova is the same way as developing native applications. However, Cordova uses WebView rendering engine to render the user interface. The mobile operating system has the most important part such as services, sensors, input and Graphics. Apache Cordova application can make use of native API to develop native-like application because Apache Cordova can access the hardware function of the mobile device. User interface rendered by WebView with the common codebase developed into hybrid apps which enables better user experience Figure 2 shows the Apache Cordova Application architecture[10]. 


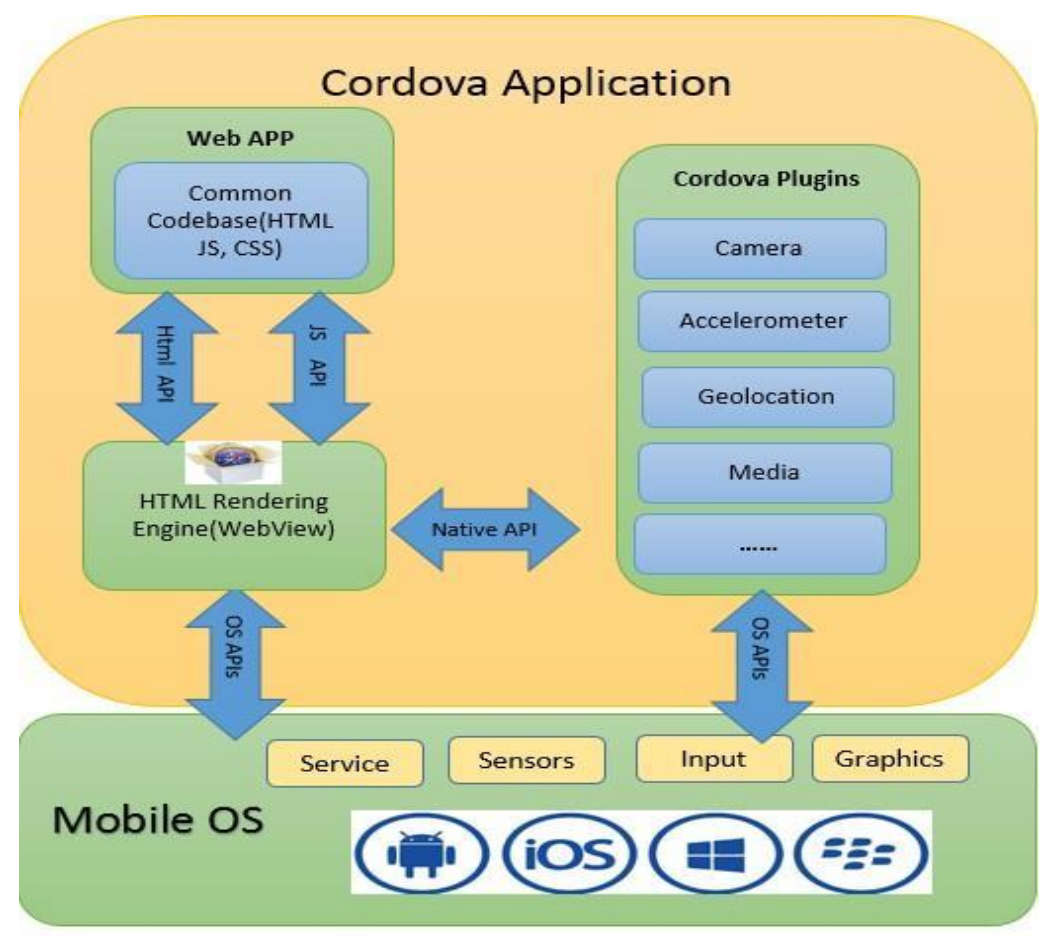

Fig. 2. Cordova Application[10]

\subsection{The developed app (I Can Talk)}

An application "I Can Talk " was developed using Cordova environment to help Autistic children to speak and express themselves via the application. The application is a tablet/mobile -based application. It has been designed according to the needs of the children specific to their category and skills. Autism spectrum is a broad spectrum with high and low disabilities. This application has been designed with techniques that have proven results on all categories such as high, medium and low Autism spectrum children. The application consists of both visual and audio aids in order to help children feel comfortable and associate objects more easily with their original meaning and connections. The application is innovative that it developed in Arabic language to serve Saudi Autistic children, as most of the autistic applications available serve English learners.

The application is designed for boys and girls where the kid chooses the gender then starts using the application. This feature personalizes the application. If a kid is a girl, a girl's voice speak and vice versa. Risks that were faced during the implementation of this application were finding images needed to be gathered, the voice needed to be added to the words. We need a kid's voice (girl and a boy) to make the application more into a voice of kid narrated by a kid. 
In the coming section detailed interfaces presented to get a better understanding of the developed application.

Figure 3 shows the main application interface where kid selects the gender to start the application. Choosing a boy the application content narrated by a boy's voice, while choosing a girl content narrated by girl's voice. Figure 3 shows the boy's main interface where he chooses between, I Want to start expressing what he wants, I Am to express his feelings or chooses to open the paint to paint. In Appendix 2 the girl's screens are added.
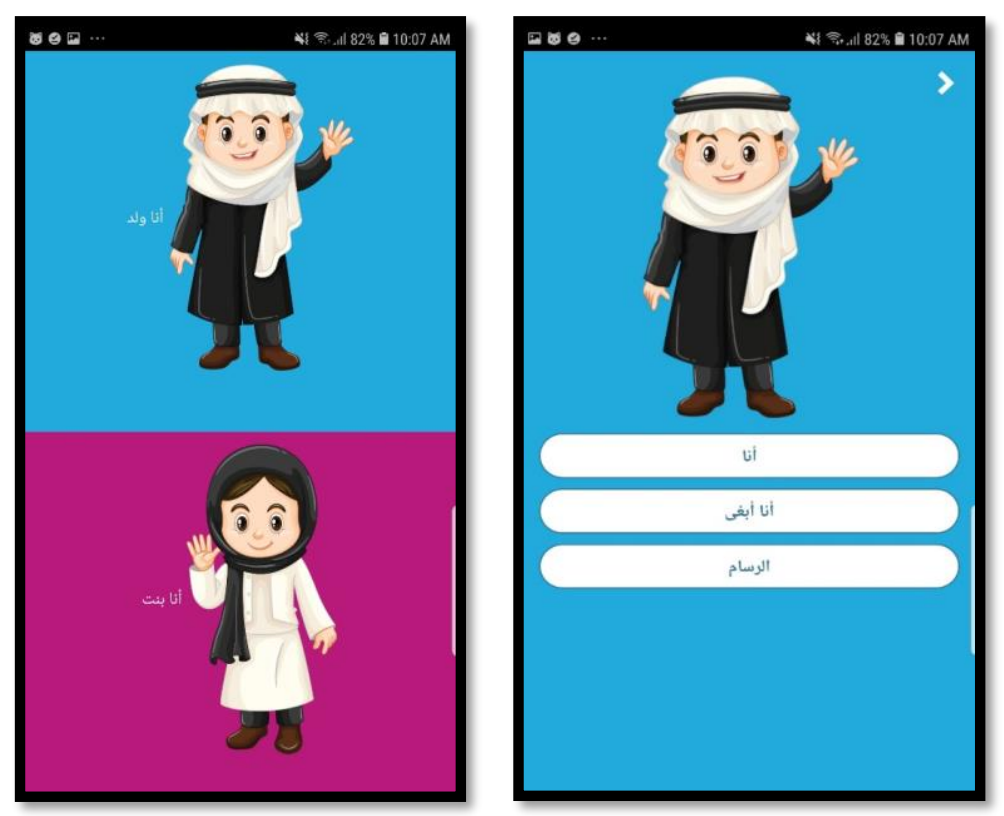

Fig. 3. Main Interface

Figure 4 presents when the kid chooses I Am there images to choose from expressing feelings as happy, angry, hungry or sad, after choosing the feeling the kid can press the play button to express the feeling with the boy's voice.

Figure 5 shows the process of forming the sentence I want to drink orange juice, where first the kid choose I Am from the main menu then the second interface show some verbs as drink, eat, play or go, after selecting the verb, the application display the next screen associated with the verb chosen, for example in the illustrated example the kid chose I want to drink, then the application displays an interface with drinks the kid chooses from, as milk, orange juice or water. In the Figure 3-4, the sentence formed was "I Want to Drink Orange Juice" as soon as the kid express his need the sentence can be played by clicking the play button, so the surrounding audience can understand what the autistic kid needs.

Figure 6 shows the paint tools, when kid click on paint from main menu, he forwarded to the following screens where he chooses to view the tools, choose the color, 
the brush width and start painting. Furthermore, the kid can choose the size of the brush.

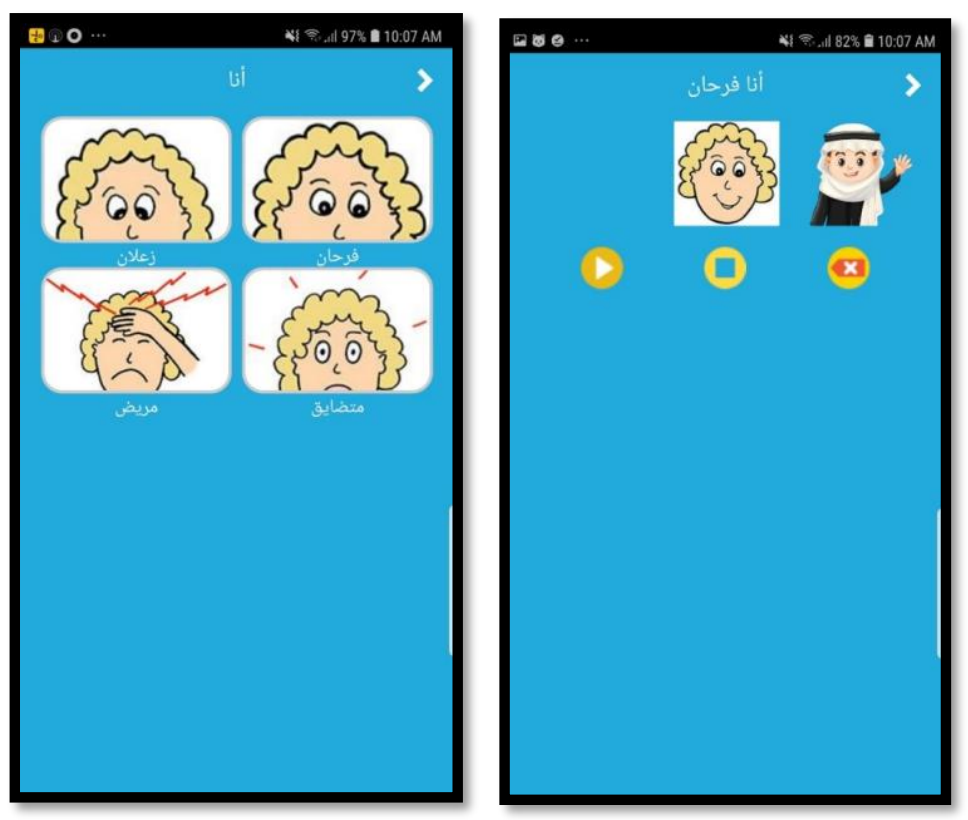

Fig. 4. I Am Happy
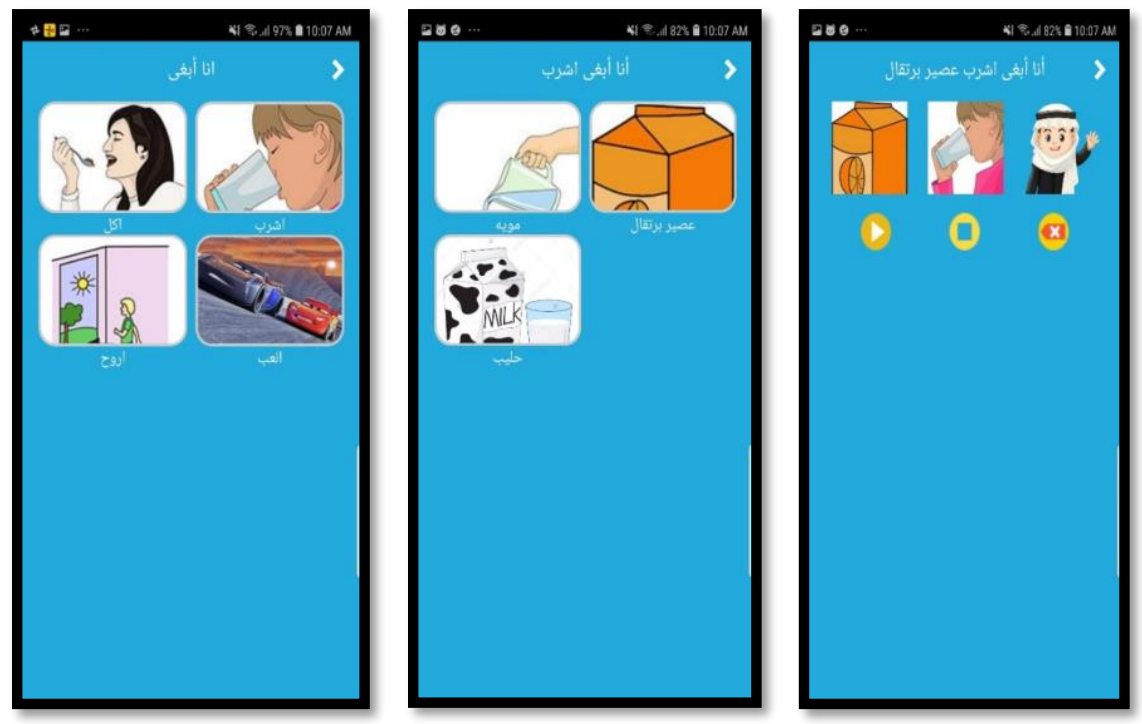

Fig. 5. I want to Drink Orange Juice 

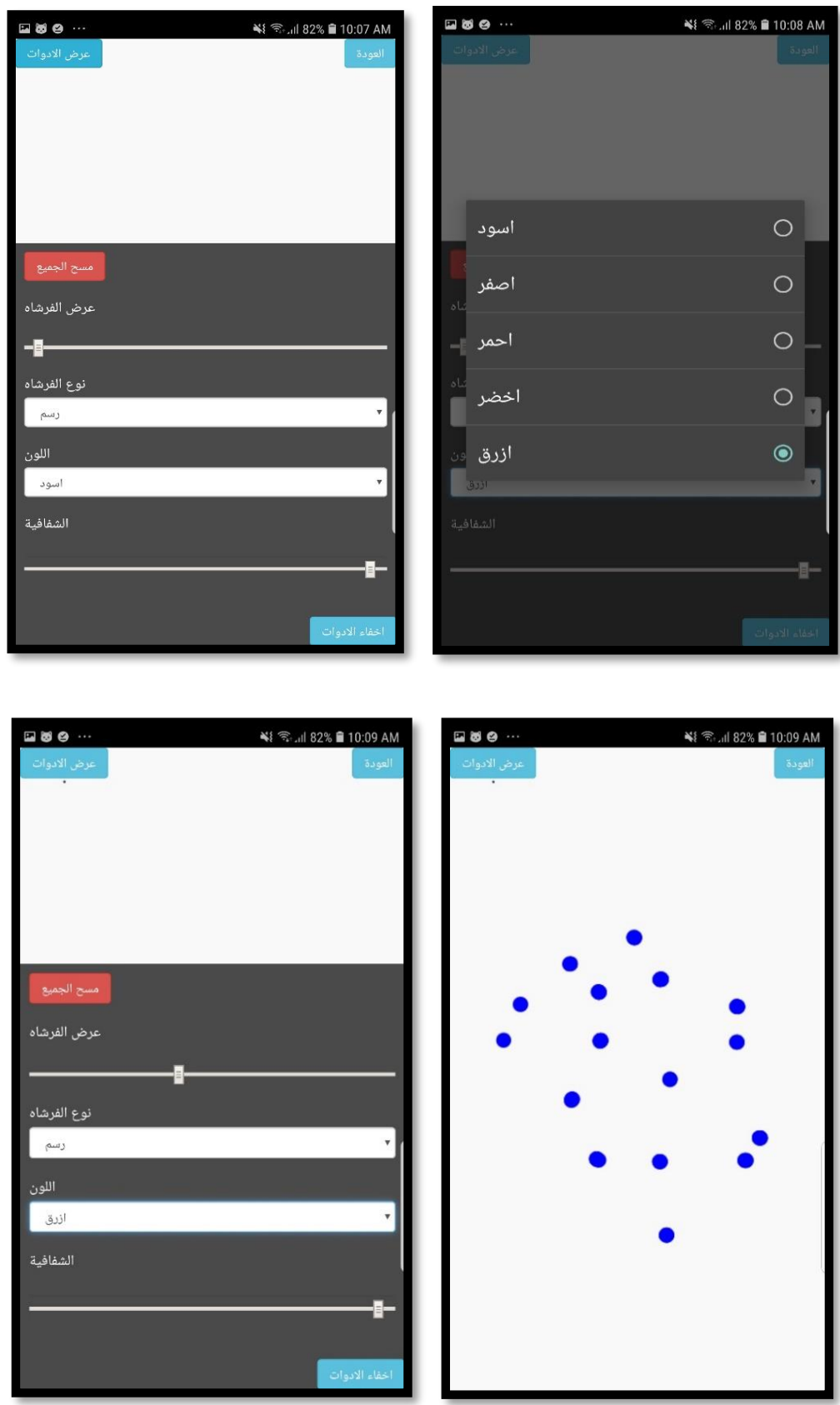

Fig. 6. Paint Application

The system development was undertaken to observe and answer the problems relevant to arising high speed Autism syndrome and for children with speech disabilities. 
The application developed is a free application for all users and has been developed for good purposes. The application has been constructed, designed and developed according to the market standards and clinical standards that need to be observed. The constructed application allows users with disabilities in speech to communicate in a much effective manner and express their needs more clearly. It also helps them in associating meaning to the objects that have been clearly defined and constructed. The problem that needed to be addressed included the speech delay and disabilities for children. This also presented a challenge to develop an application that would suffice their needs and help them learn more and thus easily communicate in their normal life besides their obvious disability due to the speech delay or due to the autism syndrome. The application developed has presented very high claims and high efficiency values for the outputs and outcomes observed. This study targets the non-speech children who can use the mobile phones and tablets. Tablets are used widely today by children because it is easy to use. The application helps to communicate with surrounding environment. This is a mobile application so we assume that autistic users are familiar with dealing with mobile devices. The constraints on this study is that it targets children who can use a tablet, also the application supports only Arabic language, finally the system is an Android/iOS application.

\section{Results and Discussion}

\subsection{Questionnaire}

A questionnaire was conducted on Google Docs, Link to the questionnaire. The questionnaire was based on Ease of use based on the simplicity to perform the tasks on the application. Feedback: rated participants' or their guardians' perspectives on the application. Satisfaction: rated how they felt about the application. Predictability: rated the ease of interpreting the images without reading the text under each one and formulating sentences using pictures only. 67 respondents responded to our survey and their answers detailed underneath, the survey included the link the application to download for teachers/parents for Autistic children to answer some questions about the application. Questions were designed to fit all the attributes of the (M-UTUAT) Model and in this section a detailed analysis of the questions presented.

Ages of autistic children using I Can Talk application were $64.2 \%$ between 4-6 years old followed by $29.9 \%$ aged between $7-9$ and then only $6 \%$ ranged between 10 12 years old as shown in Table 1. Girls were $56.7 \%$ from the total experimenters and rest were boys. 
Table 1. Sample Profile

\begin{tabular}{|l|c|c|c|}
\hline Measure & Value & Frequency & Percentage \\
\hline \multirow{4}{*}{ Age } & $1-3$ & 0 & 0 \\
& $4-6$ & 43 & 64.2 \\
& $7-9$ & 20 & 29.9 \\
& $10-12$ & 4 & 6.0 \\
\hline \multirow{3}{*}{ Gender } & $13-15$ & 0 & 0 \\
\cline { 2 - 4 } & Total & $\mathbf{6 7}$ & $\mathbf{1 0 0}$ \\
\cline { 2 - 4 } & Boy & 38 & 56.7 \\
& Girl & 29 & 43.3 \\
\cline { 2 - 4 } & Total & $\mathbf{6 7}$ & $\mathbf{1 0 0}$ \\
\hline
\end{tabular}

\section{2 (PACMAD) Testing results}

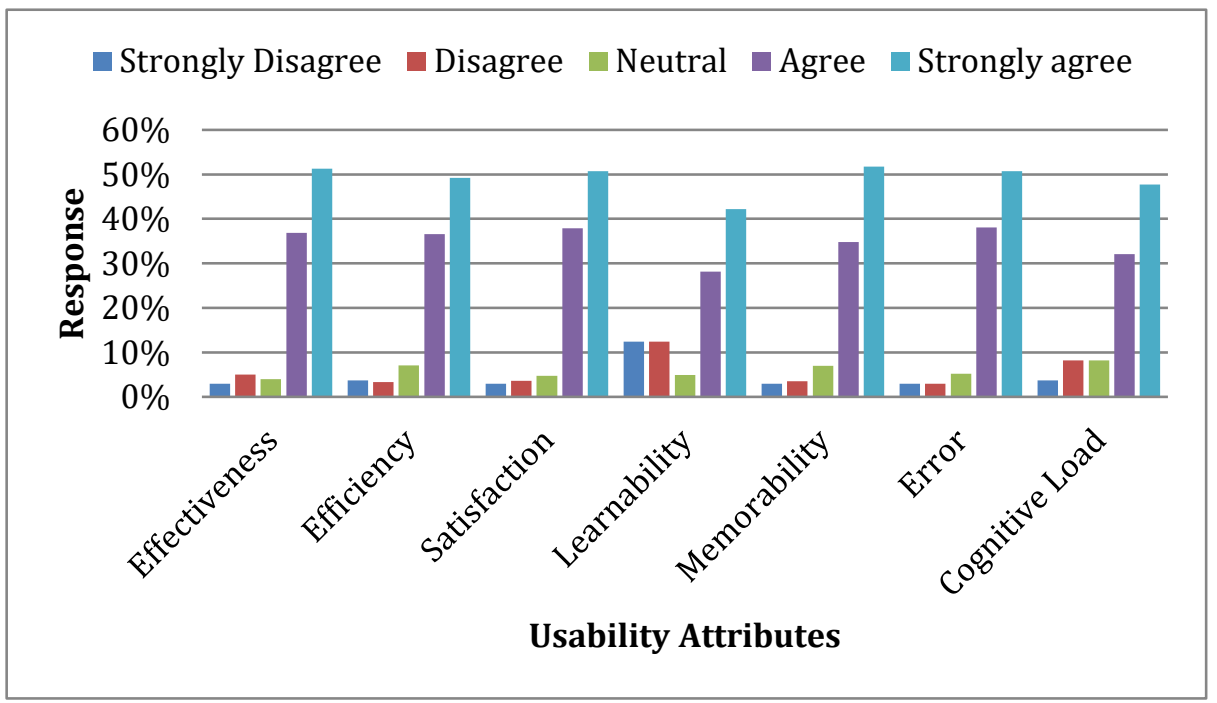

Fig. 7. Respondent's Perception on the usability of the App

Effectiveness: According to the effectiveness part in Figure 7, 37\% of respondents agree and $51 \%$ strongly agree, with total of $88 \%$, this is above the average and means that respondents are satisfied with the effectiveness part. $4 \%$ of respondents consider effectiveness of developed mobile application as neutral. 5\% disagree and 3\% strongly disagreed with effectiveness of (ICT). High percentage of agreement on Effectiveness attribute indicates that (ICT) helped to improve communication between autistic kids and teacher/parent. In addition, (ICT) helped autistic kids to develop sentences and express their selves. Also having different narrators as boys and girls was effective and beneficial.

Efficiency: $37 \%$ of respondents concur and $49 \%$ strongly concur in response to efficiency questions. Meaning that $86 \%$ of respondents consider it above the average level. Some $7 \%$ are between strongly disapprove and disapprove with this part. The efficiency attribute of the (ICT) was agreed by a high percentage of respondents. This 
means that the architecture of (ICT) is simple, and the terminologies utilized in the application are good for autistic kids. Besides, all the objectives for learning were achieved within a short duration. Installing the app (ICT) was speedy. Respondents agreed that the screens in (ICT) gave them clear directions and thus didn't need tech support to use the app. Respondents agreed to autistic kids creating a sentence with "I am" and "I Want" on the application was easy.

Satisfaction: Satisfaction part of the questionnaire was to measure satisfaction. $89 \%$ in total between concur and strongly concur; this degree puts the application above average including $38 \%$ who disapprove and $51 \%$ who strongly disapprove. Thus, most respondents believed (ICT) transformed their way of communication of autistic kids. They showed interest in (ICT) .Respondents overall measure agrees that that the (ICT) was a good application and they would recommend it to other autistic kids.

Learnability: "Learnability" part in the questionnaire provided that $28 \%$ of the respondents concur with this application, almost $42 \%$ strongly concur and $70 \%$ as a total which is considered the application has learnability. 5\% consider learnability at an average level. $12 \%$ disapprove and $12 \%$ strongly disapprove with the learnability of application with a total of $24 \%$ which is a minimum percent. Overall, measure of the learnability shows that respondents agreed that the guides in (ICT) directed them clearly on how to use the app and they didn't need technical support to use (ICT). It was very easy to understand (ICT).

Memorability: "Memorability" part in the survey was estimated and the outcomes was $35 \%$ of respondents concur and $52 \%$ strongly concur with memorability which implies that $87 \%$ of the respondents feel that the application is over the average level in regards to memorability, $7 \%$ see (ICT) as average level dimension and just $6 \%$ of respondents think about this characteristic underneath the level. The after effects of respondent's on the memorability part of ease of use demonstrates that respondents felt it was simple for autistic children to recollect how to utilize the portable application in the following occasions, and they felt confident that they could utilize the versatile application easily in future. They trusted the utilization of pictures in the application help them to review how to utilize (ICT) effectively. This element shows high convenience of the (ICT).

Error : The results of error part was: $38 \%$ of respondents concur and $51 \%$ strongly concur with memorability, which means that $87 \%$ of the respondents consider the application is above the average level of error. These results show respondents agreed that only few kids made few errors during (ICT) use.

Cognitive load: This part of the study analyses the response of respondents as we can see $32 \%$ of respondents concur and $48 \%$ strongly concur indicating that $80 \%$ of the respondents consider the application in the aspect of cognitive load above the average level whereas $8 \%$ consider this it at average level. $12 \%$ consider the cognitive load under the average. Cognitive load concerned with the performance of users of mobile applications when performing special tasks such as walking, while using the mobile device, here we need to note that the nature of autistic kids does not allow them to do more than one thing at a time so getting that he kids can only focus on one thing is a right assumptions when dealing with autistic kids. Also, their experience 
was that (ICT) instructions were not hard to follow, so when finishing (ICT) tasks, they had low stress levels.

\subsection{UTUAT Testing results}

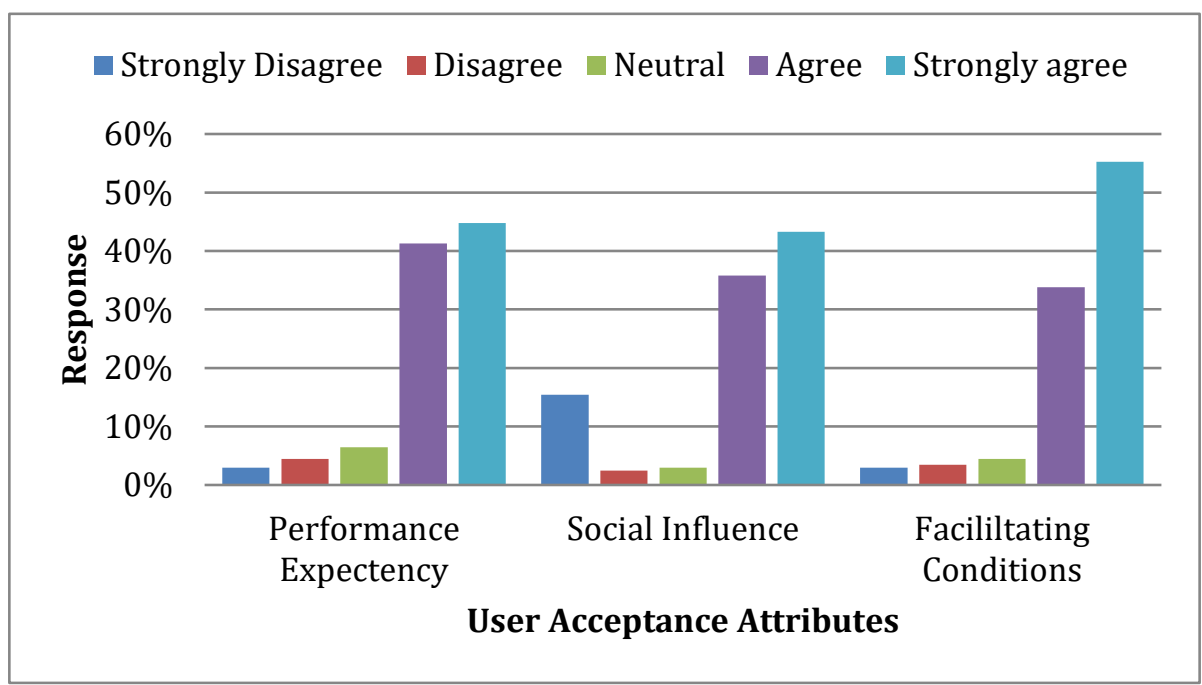

Fig. 8. Respondents Perception on the user acceptance of the App

Performance expentancy: Performance expectancy is defined as" the degree to which an individual believes that using the system helped him or her to attain gains in a job performance"[8]. Our discoveries uncover that the higher the client's desire to perform well with versatile application, the higher the social expectation to utilize it. As for the Performance expectancy, $86 \%$ of all respondents expressed that they concurred $(41 \%)$ or even emphatically concurred $(45 \%)$ using (ICT) helped the autistic kids to express themselves and create sentences. These results show that performance expectancy is positively related to the behavioral intention to use and accept (ICT).

Social infulance: Social influence defined as "the degree to which an individual perceives that important others believe he or she should use the new system "[8]Social influence may also come from, friends, family and other people who influence the behavior of autistic kid and who are important to this kid. In case that (ICT) urged by parents, teachers, therefore recommendations of friends and family to use (ICT) can be seen as more voluntary. As we see in Figure 8 total of $79 \%$ is achieved for social influence where $43 \%$ strongly agree and $36 \%$ agree.

Facilitationg conditions: This is characterized as" how much a person trusts that a specialized framework exists to help in the usage of the framework"[8] . Facilitating conditions might be accessible to a different degree in comparison to others. For example (ICT) is a free application that is really an important facilitating condition, besides having internet to download the application and use it is an important factor in using (ICT). Lack of facilitating conditions may lead to barriers and, conse- 
quently, the intentions of having users to use (ICT) is expected to be lower. The results of facilitating conditions was $89 \%$ with $55 \%$ strongly agree followed by $34 \%$ agreed to the facilitating conditions questions.

\subsection{Model validation using cronbach's alpha}

Cronbach's alpha[11] is an internal consistency (reliability) measure, explaining, how items are as a group. It is known as the measure of scale reliability. A "high" value for alpha does not imply that the measure is unidimensional. If, in addition to measuring internal consistency, an evidence is provided that the scale in question is unidimensional, additional analyses can be performed. Exploratory factor analysis is one method of checking dimensionality. Cronbach's alpha is not a statistical test - it is a coefficient of reliability (or consistency).

Cronbach's alpha can be written as a function of the number of test items and the average inter-correlation among the items. Below, for conceptual purposes, we show the formula for the Cronbach's alpha:

$$
\alpha=\frac{N \cdot \bar{c}}{\bar{v}+(N-1 \cdot \bar{c})}
$$

Here $\mathrm{N}$ is equal to the number of items, c-bar is the average inter-item covariance among the items and v-bar equals the average variance.

From formula above it can be stated that if the number of items increase, Cronbach's alpha increases too. Additionally, if the average inter-item correlation is low, alpha will be low. As the average inter-item correlation increases, Cronbach's alpha increases as well. Table 2 shows the Cronbach's alpha Consistency.

Table 2. Cronbach's alpha Consistency

\begin{tabular}{|l|l|}
\hline \multicolumn{1}{|c|}{ Cronbach's alpha } & \multicolumn{1}{c|}{ Internal consistency } \\
\hline $0.9 \leq \alpha$ & Excellent \\
\hline $0.8 \leq \alpha<0.9$ & Good \\
\hline $0.7 \leq \alpha<0.8$ & Acceptable \\
\hline $0.6 \leq \alpha<0.7$ & Questionable \\
\hline $0.5 \leq \alpha<0.6$ & Poor \\
\hline$\alpha<0.5$ & Unacceptable \\
\hline
\end{tabular}




\section{5 (PACMAD) Validation using Cronbach's alpha}

Table 3. (PACMAD) Validation using Cronbach's alpha

\begin{tabular}{|l|l|}
\hline \multicolumn{1}{|c|}{ Attribute } & \multicolumn{1}{c|}{ Value } \\
\hline Effectiveness & 0.88 \\
\hline Efficiency & 0.89 \\
\hline Satisfaction & 0.91 \\
\hline Learnability & 0.79 \\
\hline Memorability & 0.87 \\
\hline Error & 0.82 \\
\hline Cognitive Load & 0.65 \\
\hline
\end{tabular}

As shown from Table 3, (PACMAD) validation using Cronbach's alpha .The alpha coefficient for the Effectiveness is .88 , Efficiency is 0.89 , Memorability is 0.87 and Error is 0.82 , we can say that these four attributes have good alpha suggesting that the items have relatively high internal consistency. Satisfaction is 0.91 which is excellent, Learnability is 0.79 which is acceptable . Regarding Cognitive Load the measure was 0.65 .

\section{6 (UTAUT) Validation using Cronbach's alpha}

Table 4. (UTAUT) Validation using Cronbach's alpha

\begin{tabular}{|l|c|}
\hline \multicolumn{1}{|c|}{ Attribute } & Value \\
\hline Performance Expectancy & 0.89 \\
\hline Social Influence & 0.58 \\
\hline Facilitating Conditions & 0.92 \\
\hline
\end{tabular}

As shown from Table 4, UTUAT validation using Cronbach's alpha. The alpha coefficient for the Performance Expectancy is .89 which is good, and Facilitating Conditions is 0.92 which is an excellent value which means that its more reliable as per Cronbach's alpha. As for Social Influence, the value we received was 0.58 . Well, this number is considered poor. We believe that there are two main reason for having this low value. First, the number of questions in social influence is only three. Cronbach's alpha value is known to be a function of the number of questions. So, the less questions normally the smaller the alpha value. Second, after reviewing the questions related to social influence and asked several people to review the, it turns out that these questions contain some vagueness which might cause a confusion for participants when they answered them.

\subsection{Discussion}

The proposed model in this study (M-UTUAT) evaluation of mobile learning application (ICT), includes 7 (PACMAD) attributes and three UTUAT factors that affect the whole usability evaluation model. Results of this study indicate that the proposed 
mobile application (ICT) provides an efficient and effective mobile learning mechanism by improving the autistic kid's skills as detailed in the previous section. (PACMAD) factors results were as follows effectiveness part nearly $88 \%$, efficiency results was $86 \%$, satisfaction was $89 \%$, memorability was $87 \%$ and cognitive load was $80 \%$, from all previous results stated we concluded that (ICT) was above average in effectiveness, efficiency, satisfaction, memorability, and cognitive load. Learnability was $70 \%$ as a total which is an average. UTUAT Performance expectancy was $86 \%$. Social influence was a total of $79 \%$ is achieved for social influence. Facilitating conditions was $89 \%$.

\section{Conclusion and Future Works}

The accompanying remarkable points rose up out of the discoveries and dialog displayed in this paper. First, we utilized the UTUAT and (PACMAD) models in this paper and after utilization and research we developed our own model (M-UTUAT). Second, an application (ICT) was tailored for this research to overcome the bugs in the autistic kids' applications available in the market by developing an application tailored for Saudi autistic kids with the narration of a boy and a girl. Third, a questionnaire was distributed to test our new model (M-UTUAT) and results was detailed in previous chapter. Fourth, Cronbach's alpha measure was used to measure the (MUTUAT) test conducted. Results of this study indicate that the proposed mobile application (ICT) provides an efficient and effective mobile learning mechanism by improving the autistic kid's skills as detailed in the previous section. This paper aims to evaluate is the process of gathering information about the worth of (ICT) application designed for autistic kids for the purpose of improving the application or making decisions about effectiveness of the application. Evaluation is concerned with understanding how the processes of learning can be enhanced, transformed, to meet the criteria it was developed for. This paper focuses on the usability issues of smart phone application (ICT) designed for people suffering from (ASD). Usability of I CT applications is analyzed on the basis of a questionnaire conducted on the (ASD) patients.

Several improvements related to technical and non-technical specifications of the developed mobile applications, the developed (UTAUT) model, and the conducted survey that can be done which are listed in the below sub sections. For instance, we have received many comments from the questionnaire participants to extend the application with more features to make it more usable. We believe that having limited features for the application have an impact on the questionnaire results. So, the below list here can be developed to increase the usability of the application: Increase the localization of the application i.e. include additional accent based on the region or country, Improve the interactivity of the application by using some sort of virtual assistant like Avatars, Include gamification to the applications and Provide activity tracking feature in the app to be able to study the behavior of the children while using the application.

In addition, there are more advanced ways of conducting the survey and collect results. We believe that the first impression has a big impact on answering the question- 
naire questions. These advanced ways require more time and more effort and heavily depends on the availability of participants who are willing to volunteer until the end of the study. On-site experimental study is one way to address this concern. Having the participates located in one geographical location and asking them to use the applications to do certain tasks and then asking them to fill the survey definitely improve the accuracy and reliability of the results. This can also be extended in time. So, instead, this study can be extended for a longer period of time to allow the children to be fully immersed with the application before they or their parents can provide feedback.

\section{$5 \quad$ References}

[1] J. F. Lehman, "The diagnostic and statistical manual of mental disorders," 2000.

[2] A. Speaks, "No Title," 2019. https://www.autismspeaks.org (accessed Feb. 01, 2020).

[3] I. Chaidi and A. Drigas, "Autism, Expression, and Understanding of Emotions: Literature Review,” Int. J. Online Biomed. Eng., vol. 16, no. 02, pp. 94-111, 2020. https://doi.org/ 10.3991/ijoe.v16i02.11991

[4] "Guidance on Usability," 2020. https://www.sis.se/api/document/preview/611299/ (accessed Feb. 05, 2019).

[5] V. A. Prudnikov, "Modern Development Prospects of Programs in Engineering Education inside National University that Use Information and Communication Technologies," Int. J. Eng. Pedagog., vol. 10, no. 3, pp. 64-81, 2020. https://doi.org/10.3991/ijep.v10i3.12619

[6] H. Mohammad and F. Abu-Amara, "A Mobile Social and Communication Tool for Autism,” Int. J. Emerg. Technol. Learn., vol. 14, no. 19, pp. 159-167, 2019. https://doi.org/ $\underline{10.3991 / i j e t . v 14 i 19.10887}$

[7] T. A. S.-L.-H. Association, "Augmentative and Alternative Communication (AAC)"," 2019. http://www.asha.org/public/speech/disorders/AAC/(accessed Feb. 03, 2019).

[8] V. Venkatesh, M. G. Morris, G. B. Davis, and F. D. Davis, "User acceptance of information technology: Toward a unified view," MIS Q., pp. 425-478, 2003. https://doi. org/10.2307/30036540

[9] R. Harrison, D. Flood, and D. Duce, "Usability of mobile applications: literature review and rationale for a new usability model," J. Interact. Sci., vol. 1, no. 1, p. 1, 2013. https:// doi.org/10.1186/2194-0827-1-1

[10] Q. Lan, X. Chen, G. Song, and H. Parsaei, "Developing Cross-platform Mobile Applications for Real-Time Remote Experiment," in E-Learn: World Conference on E-Learning in Corporate, Government, Healthcare, and Higher Education, 2015, pp. 1808-1815.

[11] "UCLA Institute of Digital Research and Education," 2019. https://stats.idre.ucla.edu/ spss/faq/what-does-cronbachs-alpha-mean/ (accessed Feb. 03, 2019).

\section{Authors}

Mohammed Fouad Hajjaris member of Taibah University. He works in the position of Computer Technician at the College of Computer Science and Engineering. 
Saad Talal Alharbi is member of Taibah University. Faculty member works as a professor in the Department of Computer Science.

Fahad Mahmoud Ghabban is member of Taibah University. Faculty member works as an assistant professor in the Department of Information Systems. He works in the position of Vice Dean for Postgraduate Studies and Scientific Research.

Article submitted 2020-11-16. Resubmitted 2021-01-20. Final acceptance 2021-01-20. Final version published as submitted by the authors. 